\title{
Factors determining the success of radiofrequency denervation in lumbar facet joint pain: a prospective study
}

\author{
Konrad Streitberger • Tina Müller • \\ Urs Eichenberger $\cdot$ Sven Trelle $\cdot$ Michele Curatolo
}

Received: 27 January 2011/Revised: 31 May 2011/ Accepted: 12 June 2011/Published online: 30 June 2011

(c) Springer-Verlag 2011

\begin{abstract}
Background and aims Radiofrequency denervation (RF) of the lumbar facet joints has been shown to be effective in well-selected patients. However, long-term success varies between studies. We evaluated the influence of selected psychosocial and constitutional factors on the outcome of $\mathrm{RF}$, expressed as the duration of pain relief.

Methods This prospective observational study included 44 patients who received RF denervations at the University Hospital of Berne. Success was defined as at least 50\% pain reduction 7-21 days, 6 months and 1 year after RF therapy. The Cox-regression analysis was performed to evaluate the influence of the following factors on the duration of success: age, sex, depression, work inability and previous surgery.

Results Complete follow-up was available for 41 patients. The success rate 7-21 days after the denervation was $76 \%$. It decreased to $32 \%$ at 6 months and to $22 \%$ at 1 year. The median success duration was 17 weeks $(95 \%$ CI 10-26). The Cox-regression analysis showed a significant shorter duration of success for patients with depression (hazard ratio [HR] 2.97, 95\% CI 1.32-6.65), previous surgery (HR 2.39, 95\% CI 1.10-5.21) and number of treated joints (HR 1.95 for each increase in the number of joints, 95\% CI 1.14-3.33). In bivariate analyses, only depression was kept to be significant.
\end{abstract}

K. Streitberger $(\bowtie) \cdot$ T. Müller · U. Eichenberger · M. Curatolo Department of Anaesthesiology and Pain Therapy, University Hospital of Bern, Inselspital, 3010 Bern, Switzerland

e-mail: konrad.streitberger@insel.ch

S. Trelle

Clinical Trial Unit Bern, Bern University Hospital, Bern,

Switzerland
Conclusions Depression seems to be related with a short duration of success. Based on these findings, a comprehensive study is warranted to evaluate whether psychosocial factors have to be considered when recruiting patients for radiofrequency denervation.

Keywords Radiofrequency - Lumbar facet joint . Low back pain - Outcome factors

\section{Introduction}

One of the possible causes of low back pain is pathology of the facet joints. The prevalence of pain originating from the facet joint is unclear, ranging from 10 to $40 \%$ in patients with axial low back pain $[1,2]$. A diagnostic infiltration with local anesthetics of the two supplying medial branch nerves confirms that the pain has its origin in the facet joint [3]. Radiofrequency (RF) denervation of the supplying medial branch nerves is a valuable treatment, whose evidence was judged as moderate for shortand long-term relief in a systematic review [4]. Studies that selected patients by controlled medial branch blocks and used a meticulous RF technique have shown success rates of $43-87 \% 1$ year after RF therapy [5]. In most studies, patients with prior low back surgery were excluded and the influence of depression on the results was not evaluated.

In clinical practice, conditions frequently differ from those encountered in the setting of research. Studies tend to use strict inclusion criteria, e.g., excluding patients with previous surgery, psychosocial disorders, compensation claims, major radiological changes of the spine, etc. As a result, data from these studies may not be applicable to clinical practice, whereby such factors are frequently 
not considered as exclusion criteria for interventional treatments.

Only a few studies evaluated psychosocial factors influencing the diagnosis or treatment in facet joint pain. A post hoc analysis of two randomized controlled studies showed that psychologically vulnerable patients tend not to respond to radiofrequency treatment, suggesting that psychological factors may be important for the treatment's success [6].

The aim of our prospective observational study was to evaluate if the success of RF facet joint denervation is influenced by factors frequently encountered in clinical practice: depression, work inability, consumption of opioids, previous surgery and numbers of treated joints.

\section{Materials and methods}

From January 2006 to June 2008, consecutive patients receiving RF denervation at the Department of Anaesthesiology and Pain Therapy of the University Hospital of Berne were included in a prospective 1-year follow-up. Inclusion criteria were chronic low back pain of at least 1 year with or without non-radicular pain radiation into the legs, a positive diagnosis of facet joint pain (see below) and age older than 18 years. Exclusion criteria were radicular pain (defined as neurological findings of nerve root dysfunction with typical radiation of pain), bleeding diathesis, pregnancy, infection, implanted electrical devices, and insufficient knowledge of German, French or Italian language.

Written informed consent to receive the treatments described below and to use the data for analysis and publication in an anonymous form were obtained from all participants. The study was approved by the hospital institutional board.

\section{Diagnostic procedure}

The intensity of pain was assessed using a $10 \mathrm{~cm}$ visual analog scale (VAS) from 0: no pain to 10: worst imaginable pain. VAS was recorded before the diagnostic block and 30, 60, 90 and $120 \mathrm{~min}$ after injection of the last dose of local anesthetic. Additionally, to estimate the improvement of function, patients were asked before the block to indicate which spine movements were mostly restricted due to pain and if those improved substantially after the injection.

The diagnostic blocks were performed under fluoroscopic control according to validated techniques [7]. The joints to be tested were selected depending on the areas of pain (side and segment) uni- or bilaterally. The selected joints were recognized radiologically by counting caudally from the first lumbar vertebra as well as cranially from the sacrum.

For two subsequent blocks at two different days, we used lidocaine $2 \%$ and bupivacaine $0.5 \%$ in a crossover randomized double blind fashion. Controlled blocks are recommended because of a $25-41 \%$ false positive response when using only single blocks [8]. The local anesthetic injected at the first session was assigned randomly by drawing lots. In case of a positive response to the first block, a second block was performed on the same nerves on a different day using the other local anesthetic. A block was defined to be positive when $80 \%$ pain reduction was observed no later than $30 \mathrm{~min}$ after injection, lasting more than $45 \mathrm{~min}$ and $2 \mathrm{~h}$ after lidocaine and bupivacaine injection, respectively [7]. Patients were included when both blocks were positive or when one block was positive and the other one resulted in at least $50 \%$ pain reduction with substantially improved function.

\section{Radiofrequency denervation}

Radiofrequency denervation was performed according to International Spine Intervention Society (ISIS) practice standards $[5,7,9]$ under fluoroscopic guidance at the same nerves as the diagnostic blocks.

A $22 \mathrm{G}$ spinal needle was placed at the target medial branch in an approximately $20^{\circ}$ oblique fluoroscopic view. This needle was needed to anesthetize the nerve with lidocaine $2 \%$ and to guide the placement of the cannula. We used a TCU 415 TC-Electrode into the 20G disposable cannula of $0.9 \times 150 \mathrm{~mm}$ with an active tip of $10 \mathrm{~mm}$. The electrode was connected to a HF-Koagulator Neuro $\mathrm{N}$ 50 (Stryker Leibinger GmbH, Freiburg, Germany). After applying local anesthesia of the skin, the RF-cannula was introduced in the oblique fluoroscopic view with a caudocephalad declination of approximately $20^{\circ}$ to achieve a parallel position of the electrode to the nerve. The correct position was proven in a lateral and an anterior-posterior view. A matrix of 2-5 lesions was made with a temperature of $80^{\circ} \mathrm{C}$ for $90 \mathrm{~s}$.

Patients were able to receive subsequent RF denervation if the first therapy was successful for at least 6 months; pain re-occurred with the same characteristics and a singlediagnostic block was positive.

Outcome measures and potential predictors of success

Assessments were done before, 7-21 days, 6 months and 1 year after RF denervation. They included current pain assessed by the VAS, area of pain (localized only at the back or radiating to the lower extremity), working disability (percent of working time patients had to reduce from $100 \%$ due to pain), pain medication, previous surgery 
due to pain and Beck Depression Inventory (BDI). The BDI assesses possible changes in mood and depressive tendencies. We used the German version of the BDI [10].

We pre-defined the following variables as potential predictors of outcome: gender, age, pain duration, depression (BDI $>16$ vs. $\leq 16$ ), previous back surgery, more than $50 \%$ work inability due to pain, any kind of opioid use (WHO II and III), radiating pain, and number of treated joints.

Success of the therapy was defined as at least $50 \%$ reduction of the target pain (defined as the low back pain including radiating pain if this occurred) as assessed by the VAS.

\section{Data analysis}

If patients received more than one RF treatment, only data concerning the first treatment were included in the primary analysis. Subsequent therapies were analyzed additionally to evaluate changes in the duration after previous $\mathrm{RF}$ denervation.

First, each pre-specified factor possibly influencing the success rate was analyzed by the standard Cox-regression analysis to quantify the association between the predictive variable and duration of success. A two-sided $p$ value less than 0.05 was considered statistically significant. All factors with statistically significant association with the duration of success were included in the bivariate analyses. In a secondary analysis, we also considered multiple interventions per patient to estimate the duration of success. All statistically significant factors of the primary bivariate analyses were included in the model. The approach as described by Prentice et al. [11] was used for this analysis. The proportional hazards assumption was assessed using Schoenfeld residuals and functional forms of continuous covariates using Martingale residuals. All analyses were done in Stata 10.1 (StataCorp LP, College Station, TX, USA).

\section{Results}

During the period of data collection, we tested 275 patients with diagnostic lumbar facet nerve blocks. The blocks were positive in 47 patients $(17.1 \%)$, who then underwent RF denervation. One patient came from abroad and was excluded due to the language problems. Two patients were excluded because they did not consent for complete data evaluation before the intervention. Three further patients dropped out because they did not attend follow-up assessments. Follow-up of 41 patients ( 24 males and 17 females) was therefore available for the analysis (Fig. 1). The median age of the patients was 59 years (interquartile range,

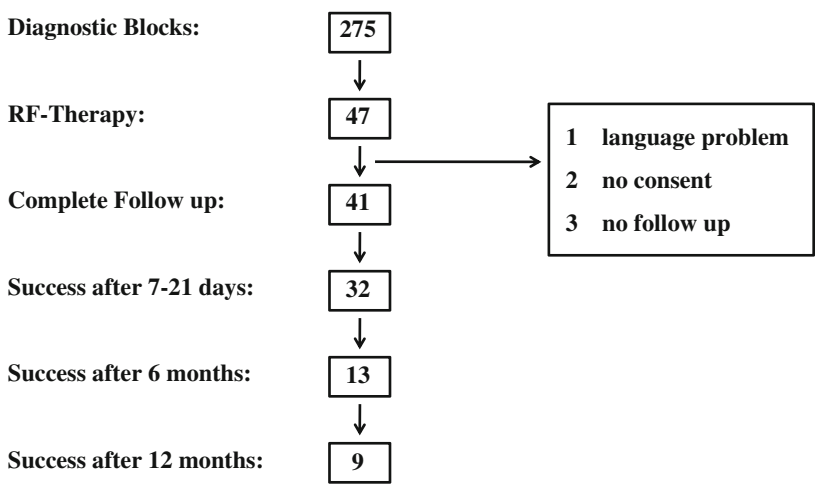

Fig. 1 Flow diagram of the included patients

IQR 48-63), the median duration of pain before the intervention was 6 years (IQR 3-10). In 10 patients treatment was performed bilaterally. Of the other 31 unilaterally treated patients 17 were treated only at one joint. Treatment included only the facet joints L3/4, L4/5 and L5/S1.

The number of successful interventions decreased from 33 (79\%) 7-21 days after RF denervation to $13(32 \%)$ at the 6 month follow-up. At 1 year, 9 of 41 patients (22\%) had at least a $50 \%$ pain reduction as compared to the baseline. The median success duration was 17 weeks $(95 \%$ CI 10-26). A complete pain relief of at least 1-year duration could be achieved in four patients $(10 \%)$.

Characterization of the patients in relation to success after 7-21 days, 6 months and 12 months is presented in Table 1. Of the nine patients with depression (BDI $>16$ ), none sustained a $50 \%$ pain reduction at 12 months and only one at 6 months. None of the seven patients with more than $50 \%$ work inability due to pain and none of the 10 patients with previous back surgery had a successful outcome at 6 months.

Median success duration of the 32 patients with BDI $\leq 16$ was 21 weeks as compared to 2 weeks in the 9

Table 1 Baseline and number of successful treated patients (VAS reduction $>50 \%$ ) at 7-21 days, 6 months and 12 months after RF therapy

\begin{tabular}{|c|c|c|c|c|}
\hline & \multirow{2}{*}{$\begin{array}{l}\text { Included } \\
\text { patients }\end{array}$} & \multicolumn{3}{|c|}{$>50 \%$ pain relief after } \\
\hline & & $7-21$ days & 6 months & 12 months \\
\hline All together & 41 & 32 & 13 & 9 \\
\hline Female & 17 & 10 & 5 & 2 \\
\hline $\mathrm{BDI}>16$ & 9 & 4 & 1 & 0 \\
\hline $\begin{array}{l}\text { Work inability } \\
>50 \%\end{array}$ & 7 & 7 & 0 & 0 \\
\hline Previous surgery & 10 & 7 & 1 & 0 \\
\hline$>1$ joint treated & 24 & 16 & 4 & 4 \\
\hline Opioids intake & 21 & 15 & 6 & 3 \\
\hline Pain radiation & 26 & 18 & 7 & 5 \\
\hline
\end{tabular}




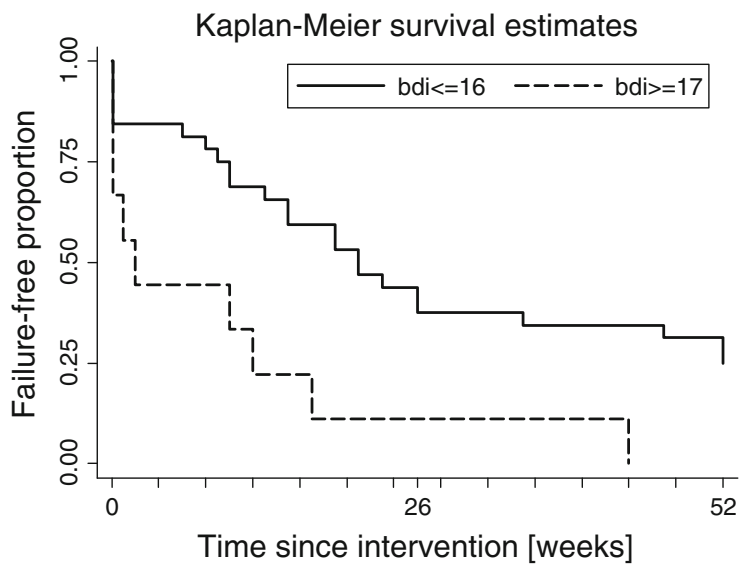

Number at risk

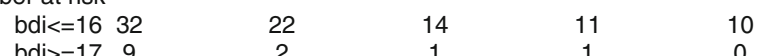

Fig. 2 Kaplan-Meier survival estimates of patients with BDI $\leq 16$ versus $\mathrm{BDI} \geq 17$

patients with $\mathrm{BDI}>16(p=0.008)$. The Cox-regression of the different potential predictors showed a statistically significantly shorter success of patients with $\mathrm{BDI}>16$ (HR 2.97, 95\% CI 1.32-6.65), previous surgery (HR 2.39, 95\% CI 1.10-5.21), and with increasing number of treated joints (HR 1.95 for each increase in the number of joints, 95\% CI 1.14-3.33) (Fig. 2; Table 2). In bivariate analyses, depression remained as the only statistically significant predictor.

The median VAS decreased from baseline of 5.6 (IQR 4.1-7.4) to 1.0 (IQR 0-2.5) 7-21 days after the intervention.

Five patients had a second and one patient two-repeated RF denervations because of the recurrence of pain (Table 3). A long-lasting effect of the first therapy could be obtained in four of these five patients. Two patients with no

Table 2 Influence of different factors on duration of success (50\% reduction in pain as compared to baseline), expressed in weeks

\begin{tabular}{llll}
\hline Factor & $\begin{array}{l}\text { Hazard } \\
\text { ratio }\end{array}$ & $95 \%$ CI & $p$ value \\
\hline Female & 1.71 & $0.86,3.4$ & 0.12 \\
Age (years) & 1.00 & $0.97,1.04$ & 0.94 \\
Pain duration (years) & 1.00 & $0.96,1.03$ & 0.94 \\
Number of treated joints & 1.95 & $1.14,3.33$ & 0.02 \\
Previous surgery (vs. no & 2.39 & $1.09,5.21$ & 0.03 \\
$\quad$ surgery) & & & \\
Opioid use (vs. no use) & 1.44 & $0.72,2.89$ & 0.30 \\
Radiating pain & 1.22 & $0.60,2.49$ & 0.58 \\
Work inability $>50 \%$ & 1.96 & $0.82,4.68$ & 0.13 \\
Depression (BDI $>16)$ & 2.97 & $1.32,6.65$ & 0.01 \\
\hline
\end{tabular}

Age, pain duration, and number of joints were included as continuous covariates with the hazard ratio relating to an increase of one unit
Table 3 Duration of $50 \%$ pain reduction (in weeks) for patients receiving more than one radiofrequency treatment $(\mathrm{RF})$

\begin{tabular}{|c|c|c|c|}
\hline & \multicolumn{3}{|c|}{$\mathrm{RF}$} \\
\hline & 1 & 2 & 3 \\
\hline Patient 1 & 21 & 40 & $>52$ \\
\hline Patient 2 & 26 & 25 & \\
\hline Patient 3 & 52 & 46 & \\
\hline Patient 4 & 47 & $>52$ & \\
\hline Patient 5 & $0^{\mathrm{a}}$ & 0 & \\
\hline Patient 6 & 13 & 0 & \\
\hline Patient 7 & $1^{\mathrm{b}}$ & $>52$ & \\
\hline
\end{tabular}

${ }^{\text {a }}$ The first RF therapy was described as very difficult because of osteophytes, therefore it was repeated after success failure. However, it resulted again in failure

b The first RF therapy was only positive for the right side, after repeating the left side the therapy was successful

effect were re-treated because incomplete nerve destruction was considered as a possible reason for the failure. One of those treatments was successful. The effect of depression was robust when repeated interventions were considered in a Cox-regression (HR 2.97, 95\% CI 1.44-6.13).

No serious adverse events related to the RF therapy were reported.

\section{Discussion}

According to results of several clinical trials, RF denervation of lumbar facet joints seems to be a very effective therapy with a success rate of $43-87 \%$ after 1 year $[7,12$, 13]. Compared to those trials, $22 \%$ success rate of our study in 1 year after therapy is disappointing. On the other hand, a recent review of randomized controlled trials showed that RF denervation of lumbar facet joints is more effective for pain relief than placebo in the short-term follow-up, but not in intermediate- or long-term follow-up, with a low evidence grade [14]. Other reviews of RF denervation discussed methodological and technical shortcomings of most of the included studies [4, 5]. Possible explanations for a worse outcome of our study, compared to the previous trials, include incorrect denervation technique, false positive diagnostic blocks or the use of different inclusion criteria.

We used a validated RF technique [5], which was also used in the study with the highest success rate [7]. We performed up to five multiple lesions in a parallel position to the nerve to assure denervation along a sufficient length of the nerve [15]. Therefore, it is unlikely that the RF technique itself or its application is the reason for the observed success rate. 
False positive blocks could be the result of placebo response. To reduce this risk, we performed double blind controlled medial branch blocks. However, compared to the ISIS criteria with at least $80 \%$ pain reduction in both blocks, we accepted $50 \%$ reduction in one of the two blocks, if there was a remarkable improvement in function, and the other block resulted in an $80 \%$ pain reduction. Furthermore, we could not evaluate the full duration of every single block. Therefore, we may have included cases with discordant responses, i.e., in which lidocaine resulted in a longer action than bupivacaine. Later analyses of the false positive rates of diagnostic blocks revealed that for lumbar RF denervations, very strict criteria are mandatory for minimizing the false positive responses of the blocks. Indeed, the lower the prevalence of a condition, the stricter the criteria for a positive diagnosis need to be in order to minimize false positive responses [8]. Thus, one possible explanation for the limited success rate is that our inclusion criteria still were not strict enough, given a prevalence of only $17 \%$ in our sample.

In contrast to the other trials, we had restricted our selection criteria only to the diagnosis of facet joint pain based on two comparative diagnostic blocks, regardless of the psychosocial conditions or previous surgery. Our aim was to evaluate if such factors may influence the long-term results of RF therapy. Based on the result, this could be an explanation for the low success rate after 1 year. This view is supported by the analysis of two previous studies. The highest long-term success rate $(87 \%)$ was achieved by a study which included only patients with a BDI $<17$ and without previous surgery [7]. In a more recent larger study, in which patients with previous surgery were excluded, the success rate decreased much faster within 1 year to $47 \%$ [12]. In this study, the influence of depression on the results was not evaluated.

The Cox-regression analysis in our study showed that the duration of success was reduced significantly by depression, previous surgery and number of denervated joints, but not by age, gender, pain duration, taking opioids or work inability of more than $50 \%$. In the bivariate control analyses, only depression was kept to be a significant predictor for the duration of pain relief. This finding is consistent with the results of a recent study on predictors for outcome of medial branch blocks with corticosteroids [16]. The authors found high levels of depression and anxiety, but no other psychosocial or medical variables, to predict poor response. Accordingly, a post hoc analysis of two randomized controlled studies showed that psychologically vulnerable patients tend not to respond to RF treatment [6]. Our results further highlight the negative influence of depression on the long-term effect of RF denervation. The mechanisms for the negative influence of depression are unclear. A possible explanation might be offered by neuroimaging studies [17].
In patients with rheumatoid arthritis, fMRI showed that depressive symptoms were related to the cerebral processing of joint pain in the medial prefrontal cortex without relation to joint inflammation [18]. This brain region seems to contribute to the maintenance and exacerbation of pain and might alter the effect of elimination of peripheral nociceptive input by RF denervation.

Previous back surgery was reported as a predictor of RF failure, in two studies $[19,20]$. Those findings were in contrast with other studies, where surgery did not influence the results [21, 22]. According to our first analysis, previous surgery seemed to be a negative predictor, but the bivariate analysis showed that this was probably due to depression as comorbidity. Also the number of denervated joints had a negative influence only in the univariate, but not in the bivariate analysis. Other potential factors like gender, age, work inability, pain duration, opioid consumption and radiation of pain [20] did not show any influence on the duration of success of RF denervation. However, there might be much more potential factors influencing the treatment's success in chronic back pain patients including peripheral and central sensitization which should be addressed in further studies.

The high success rate within 3 weeks after the intervention might include placebo effects, which are usually of short duration. In contrast, the long-term effect of 9 from 32 patients without depression seems to be a specific effect of the denervation. In the context of otherwise therapy refractory chronic low back pain, such a result still is encouraging to use facet nerve denervation with an appropriate technique in well-selected patients as suggested by Dreyfuss [7].

Our study has a major shortcoming: it included a too low number of patients for a comprehensive analysis of predictive factors. Therefore, the results provide only partial information on the predictors of the success of $\mathrm{RF}$ denervation.

In conclusion, the results of our study suggest that depression is a predictor of poor outcome of RF denervation of lumbar facet joints. Therefore, we encourage reconsidering very carefully the indication of RF denervation in chronic low back pain patients with concomitant depression. Number of segments and previous surgery are potential negative factors. Studies with large sample sizes and comprehensive assessment of potential predictors are warranted in order to improve the selection criteria of patients undergoing RF denervation of the lumbar facet joints.

Conflict of interest None.

\section{References}

1. Cohen SP, Raja SN (2007) Pathogenesis, diagnosis, and treatment of lumbar zygapophysial (facet) joint pain. Anesthesiology 106:591-614 
2. Schwarzer AC, Aprill CN, Derby R, Fortin J, Kine G, Bogduk N (1994) Clinical features of patients with pain stemming from the lumbar zygapophysial joints. Is the lumbar facet syndrome a clinical entity? Spine 19:1132-1137

3. van Kleef M, Vanelderen P, Cohen SP, Lataster A, Van Zundert J, Mekhail N (2010) 12. Pain originating from the lumbar facet joints. Pain Pract 10:459-469. doi:10.1111/j.1533-2500.2010.00 393.x

4. Boswell MV, Colson JD, Sehgal N, Dunbar EE, Epter R (2007) A systematic review of therapeutic facet joint interventions in chronic spinal pain. Pain Physician 10:229-253

5. Bogduk N, Dreyfuss P, Govind J (2009) A narrative review of lumbar medial branch neurotomy for the treatment of back pain. Pain Med 10:1035-1045. doi:10.1111/j.1526-4637.2009.00692.x

6. van Wijk RM, Geurts JW, Lousberg R, Wynne HJ, Hammink E, Knape JT, Groen GJ (2008) Psychological predictors of substantial pain reduction after minimally invasive radiofrequency and injection treatments for chronic low back pain. Pain Med 9:212-221. doi:10.1111/j.1526-4637.2007.00367.x

7. Dreyfuss P, Halbrook B, Pauza K, Joshi A, McLarty J, Bogduk N (2000) Efficacy and validity of radiofrequency neurotomy for chronic lumbar zygapophysial joint pain. Spine 25:1270-1277

8. Curatolo M, Bogduk N (2010) Diagnostic and therapeutic nerve blocks. In: Fishman SM, Ballantyne JC, Rathnell JP (eds) Bonica's management of pain. Wolters Kluwer/Lippincott Williams \& Wilkins, Philadelphia, pp 1401-1423

9. International Spine Intervention Society (2004) Lumbar medial neurotomy. In: Bogduk N (ed) Practice guidelines-spinal diagnostic and treatment procedures. International Spine Intervention Society, San Francisco, pp 188-218

10. Hautzinger M (1991) The Beck Depression Inventory in clinical practice. Nervenarzt 62:689-696

11. Prentice RL, Williams BJ, Peterson AV (1981) On the regression analysis of multivariate failure time data. Biometrika 68:373-379

12. Gofeld M, Jitendra J, Faclier G (2007) Radiofrequency denervation of the lumbar zygapophysial joints: 10-year prospective clinical audit. Pain Physician 10:291-300

13. van Kleef M, Barendse GA, Kessels A, Voets HM, Weber WE, de Lange S (1999) Randomized trial of radiofrequency lumbar facet denervation for chronic low back pain. Spine 24:1937-1942
14. Henschke N, Kuijpers T, Rubinstein SM, van Middelkoop M, Ostelo R, Verhagen A, Koes BW, van Tulder MW (2010) Injection therapy and denervation procedures for chronic lowback pain: a systematic review. Eur Spine J 19:1425-1449. doi: 10.1007/s00586-010-1411-0

15. Bogduk N, Macintosh J, Marsland A (1987) Technical limitations to the efficacy of radiofrequency neurotomy for spinal pain. Neurosurgery 20:529-535

16. Wasan AD, Jamison RN, Pham L, Tipirneni N, Nedeljkovic SS, Katz JN (2009) Psychopathology predicts the outcome of medial branch blocks with corticosteroid for chronic axial low back or cervical pain: a prospective cohort study. BMC Musculoskelet Disord 10:22. doi:10.1186/1471-2474-10-22

17. Bingel U, Tracey I (2008) Imaging CNS modulation of pain in humans. Physiology (Bethesda) 23:371-380. doi:10.1152/physiol. 00024.2008

18. Schweinhardt P, Kalk N, Wartolowska K, Chessell I, Wordsworth P, Tracey I (2008) Investigation into the neural correlates of emotional augmentation of clinical pain. Neuroimage 40:759-766. doi:10.1016/j.neuroimage.2007.12.016

19. Cohen SP, Hurley RW, Christo PJ, Winkley J, Mohiuddin MM, Stojanovic MP (2007) Clinical predictors of success and failure for lumbar facet radiofrequency denervation. Clin J Pain Vol 23:45-52

20. van Wijk RM, Geurts JW, Wynne HJ, Hammink E, Buskens E, Lousberg R, Knape JT, Groen GJ (2005) Radiofrequency denervation of lumbar facet joints in the treatment of chronic low back pain: a randomized, double-blind, sham lesion-controlled trial. Clin J Pain 21:335-344

21. North RB, Han M, Zahurak M, Kidd DH (1994) Radiofrequency lumbar facet denervation: analysis of prognostic factors. Pain 57:77-83. doi:0304-3959(94)90110-4

22. Pevsner Y, Shabat S, Catz A, Folman Y, Gepstein R (2003) The role of radiofrequency in the treatment of mechanical pain of spinal origin. Eur Spine J 12:602-605. doi:10.1007/s00586-0030605-0 